\title{
An Electron-Ion Collider at Jefferson lab
}

\author{
Anthony W. Thomas \\ Suite 1, Jefferson Lab, 12000 Jefferson Ave., Newport News VA 23606 USA \\ and College of William and Mary, Williamsburg VA 23187 USA

\begin{abstract}
Long term plans for the investigation of the quark and gluon structure of matter have for some time focussed on the possibility of an electron-ion collider, with the nuclear physics communities associated with JLab and BNL being particularly active. We
\end{abstract} \\ briefly outline the current thinking on this subject at Jefferson lab.
}

\section{Introduction}

As explained in the oral presentation [1, the original plans for an electron-ion collider (ELIC) at Jefferson Lab involved the construction of two figure-of-eight rings, intersecting at up to four collision points, with a proton energy of 30-225 GeV (30-100 GeV/A for ions up to $\mathrm{Pb}$ ) and electrons (and positrons) from 3 to $9 \mathrm{GeV}$ 2]. Since the construction of such a facility must await completion of the $12 \mathrm{GeV}$ Upgrade at Jefferson Lab [3], as well as the construction of FRIB, it is unlikely to begin much before the end of the next decade. The design for ELIC was appropriately ambitious for a world-leading machine that will not take data until the third decade of this millenium, with a design luminosity approaching $10^{35} \mathrm{~cm}^{-2} \mathrm{sec}^{-1}$. The exploration of the physics case for such a machine, which shares at least some common ground with the proposed eRHIC machine at BNL, involves an on-going collaboration under the heading of EIC [4, between the communities associated with both national laboratories.

Since the cost of such very high energy colliders is likely to be rather high, at Jefferson Lab considerable effort has recently gone into the design of possible staging options, which present a strong, self-contained physics case, yet have a cost comparable to that of FRIB. A multitude of novel suggestions for such a machine led to a very vigorous discussion between physicists, engineers and machine designers and the full user community at Jefferson Lab is just beginning to participate in these discussions. Nevertheless, there is already a very exciting design which currently appears to optimize the opportunities for scientific discovery while satisfying reasonable cost constraints. Everything which I shall present is the result of an impressive team effort by the group of people thanked in the acknowledgements. This new machine is currently known as the MEIC, or the medium energy electron-ion collider.

\section{MEIC Design Parameters}

As illustrated in Fig. 1, MEIC is designed to serve as the natural first stage of full ELIC construction. As far as possible the tunnel and components can be re-used in the ultimate machine, should that be built. The circumference of the machine is $634 \mathrm{~m}$, with straight sections of $150 \mathrm{~m}$. The proton energies range from 12 to $60 \mathrm{GeV}$, in collision with electrons (or positrons) taken from the CEBAF $12 \mathrm{GeV}$ Upgrade between 3 and $11 \mathrm{GeV}$. This allows for e-p collisions over an impressive range of cm energies, from $s=100$ to $2640 \mathrm{GeV}^{2}$. We also note that if it were to be essential, even the MEIC can be staged with a warm ion ring, allowing proton momenta up to $12 \mathrm{GeV} / \mathrm{c}$, being a somewhat less expensive first step.

DIS 2009 


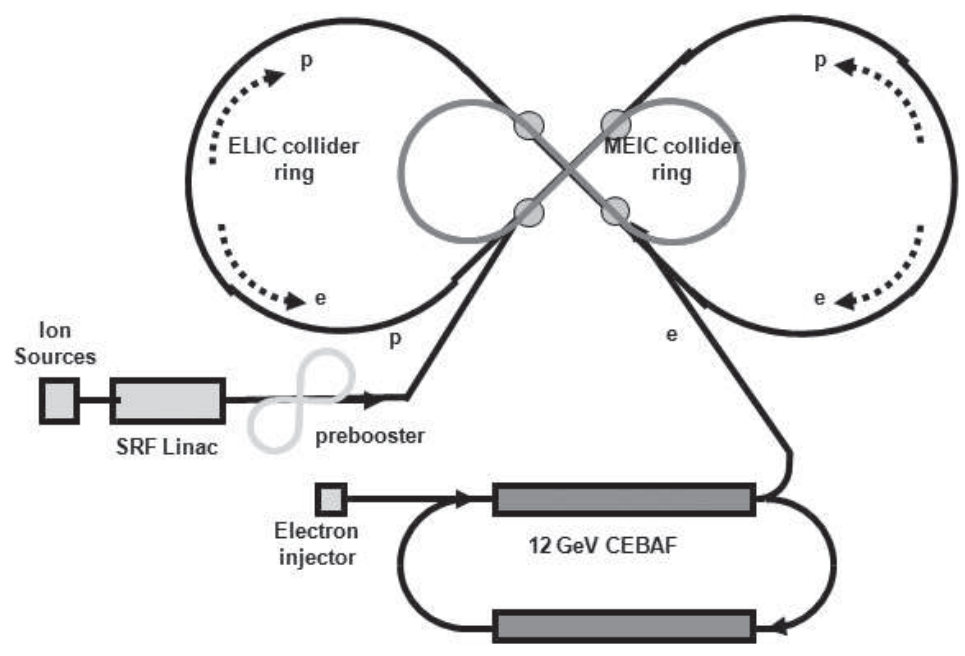

Figure 1: Layout of the ELectron Ion Collider (ELIC) and the MEIC at Jefferson Lab. Both of these machines would use polarized electrons (or positrons) at energies up to $11 \mathrm{GeV}$ from the upgrade of CEBAF which is currently underway.

As outlined earlier, a key design criterion has been to ensure that the luminosity will be appropriate for a world-leading macine in the 2020's and 30's. The current design yields excellent luminosity, around $10^{35} \mathrm{~cm}^{-2}$-sec-1, for $s \in(200,1200) \mathrm{GeV}^{2}$. The luminosity remains above $10^{33}$ over the rest of the range. In terms of physics reach, this means that one can access the structure of polarized protons at luminosities of order $10^{35}$ above $x=$ 0.0008 at $Q^{2}=1 \mathrm{GeV}^{2}$ and above $x=0.01$ at $12 \mathrm{GeV}^{2}$. For the time being, the luminosity is bounded by detector and data acquisition limitations, with the repetition rate being kept at a conservative $500 \mathrm{MHz}$. There is an urgent need for $\mathrm{R} \& \mathrm{D}$ to explore whether one could raise this rate and consequently the luminosity.

The figure of 8 structure, illustrated in Fig. 1, is designed to ensure high polarization for both the light ion and electron beams. There are four possible intersection points. It is intended to also make a polarized positron capability with the same luminosity available.

A particularly attractive feature of the MEIC design is that unlike ELIC or eRHIC, where some of the technical issues presently seem to be "very challenging" or worse, there is no issue associated with MEIC which ranks above "challenging" - with the electron cooling and travelling focussing being the two major issues. Innovative features, such as crab crossing and crab cavities seem to be almost in hand. Other important issues which need work in the near future, include beam-beam effects and the formation of the high intensity low energy ion beam but these are not regarded as especially challenging.

\section{Physics at the MEIC}

As the novel design of the MEIC is very recent, the detailed physics case matched to its unique capabilities still needs considerable work and we certainly invite all interested members of the community to join this effort. 
In many ways the proposed MEIC is the perfect complement to the $12 \mathrm{GeV}$ Upgrade currently underway at Jefferson Lab [5]. While the latter aims to define the spin and flavor dependence of nucleon and nuclear parton distribution functions in the valence region, the former is ideally suited to serve the same function for the sea. Indeed, with its capability to clinically examine the debris left from the target, this collider should enable a far deeper understanding of the origin and structure of the non-perturbative sea than we have ever been able to imagine before.

The generalized parton distributions, which will be thoroughly explored at $12 \mathrm{GeV}$ and then exploited as source of information about the distribution of angular momentum on the valence quarks [6, 7, will serve as a vital source of information on the orbital angular momentum carried by sea quarks. It remains to be seen whether they can also be used to investigate the gluon angular momentum but if a method were to be found it would be very valuable indeed. The understanding of the potential for transversity to yield information on the distribution of orbital angular momentum within the proton is at an earlier stage but there are clear indications of its potential which needs to be developed further.

As a tool to investigate the quark and gluon structure of atomic nuclei, a fundamental issue for modern nuclear physics, the MEIC offers some remarkable new possibilities. The study of the iso-vector EMC effect 8 could be dramatically advanced if one could make a comparison of the $\left(e^{-}, \bar{\nu}_{e}\right)$ and $\left(e^{+}, \nu_{e}\right)$ reactions on a variety of heavy nuclei. The ability to identify fragments of the final nucleus offers potentially novel ways to test explanations of the EMC effect. We expect the beam quality to be such that this machine should also be suitable for investigations involving parity violation, which also offers a novel look inside hadron and nucleon structure.

A number of groups have just begun to explore the potential of this machine for studying charmed systems - from the production on free nucleons, to in-medium modification to their role as a possible tool to determine the gluon angular momentum. This is an extremely promising area that merits detailed examination.

Finally, one cannot imagine constructing a machine such as MEIC, with its high level of polarization and luminosity, as well as its expanded range of invariant mass, without exploring its potential for precision tests of the Standard Model. For the present there is nothing to report in this area, but I would certainly encourage some of the younger members of the community who may actually live long enough to finish such an experiment, to begin to contemplate the possibilities.

\section{Concluding Remarks}

It is clear that one or more electron-ion colliders may well have an important role to play in the development of nuclear physics over the next few decades [9. The MEIC, which is under intense study at Jefferson Lab, represents a cost effective first stage towards the very high energy ELIC. Nevertheless, MEIC appears to have a very impressive physics program associated with it. We certainly welcome all members of the DIS community who are excited by its potential and would like to contribute.

\section{Acknowledgments}

It is a pleasure to acknowledge the tremendous amount of effort that has so far been devoted to this important project by a number of staff and users at Jefferson Lab. I would particularly

DIS 2009 
like to thank S. Bogacz, P. Chevtsov, Ya. Derbenev, R. Ent, G. Krafft, T. Horn, A. Hutton, C. Hyde-Wright, R. Li, B. Yunn, Y. Zhang, F. Klein and P. Nadel-Turonski and C. Weiss, without whom this presentation would not have been possible. This work was supported by the the U.S. Department of Energy under Contract No. DE-AC05-06OR23177, under which Jefferson Science Associates, LLC operates Jefferson Laboratory.

\section{Bibliography}

\section{References}

[1] Slides:

http://indico. cern. ch/getFile.py/access? contribId=28\&sessionId=22\&res Id=3\&materialId=slides\&conf Id=53294

[2] S. Bogacz et al., In the Proceedings of Particle Accelerator Conference (PAC 07), Albuquerque, New Mexico, 25-29 Jun 2007, pp 1935.

[3] http://www.jlab.org/div_dept/physics_division/GeV/doe_review/CDR_for_Science_Review.pdf

[4] http://web.mit.edu/eicc/

[5] A. W. Thomas, Eur. Phys. J. ST 140 (2007) 117.

[6] X. D. Ji, Phys. Rev. Lett. 78, 610 (1997) arXiv:hep-ph/9603249].

[7] A. W. Thomas, Phys. Rev. Lett. 101, 102003 (2008) arXiv:0803.2775 [hep-ph]].

[8] I. C. Cloet, W. Bentz and A. W. Thomas, arXiv:0901.3559 [nucl-th].

[9] "The Frontiers of Nuclear Science", the NSAC Long Range Plan for Nuclear Physics (2007) ; Report of the OECD Global Science Working group on Nuclear Physics, May 2008 ; IUPAP Report No. 41, "Research Facilities in Nuclear Physics" http://trshare.triumf.ca/ ${ }^{\sim}$ ramsay/IUPAP-WG9/IUPAP-Report-41.pdf 